\title{
Revisiting the link between cognitive decline and masticatory dysfunction
}

\author{
Chia-shu Lin(1)
}

\begin{abstract}
Age-related decline in cognitive functions and dementia are major challenges in geriatric healthcare. Accumulating evidence from clinical, epidemiological and animal research suggests that tooth loss may be a risk factor for the decline of cognitive functions. This issue highlights the role of the brain-stomatognathic axis in geriatric medicine. Whether input from the stomatognathic apparatus can affect the brain remains an open debate. By revisiting the evidence published in the past five years, we argue that the hypothesis regarding the association between cognitive decline and masticatory dysfunction should be carefully interpreted. Most of the available clinical and epidemiological studies present only cross-sectional data. With respect to the prospective studies, important confounding factors, such as nutritional and physical conditions, were not fully controlled for. Animal research has revealed that hippocampal deficits may play key roles in the observed cognitive decline. However, experimental intervention and outcome assessments may not capture the condition of human subjects. Brain neuroimaging research may be suitable for bridging the gap between clinical and animal research, potentially contributing to (a) the clarification of the brain network associated with mastication, (b) the identification of brain imaging markers for exploring the mechanisms underlying long-term changes in masticatory functions, and (c) the elucidation of interactions between mastication and other cognitive-affective processing systems. Three potential models of the brain-stomatognathic axis and relevant hypotheses are summarized, focusing on the sensory feedback mechanisms, the compensation of motor control, and cerebellar deficits. Finally, we highlight four critical aspects of study and experimental design that should be considered in future research: (a) the refinement of the considered behavioral assessments, (b) the inclusion of baseline changes in mental and physical conditions, (c) a prospective experimental design with longitudinal observations, and (d) a precise determination of the effect size of the association between cognitive decline and masticatory dysfunction.
\end{abstract}

\section{Background}

Does tooth loss increase the risk of dementia? Can improvements in chewing ability prevent cognitive impairment or ameliorate cognitive decline? The association between the brain and the stomatognathic system, which plays a key role in chewing and swallowing [1], has recently been hotly debated in the media [2-5]. Behind these arguments is the emerging concept of the 'brain-stomatognathic axis', generally defined as a complex communication network between the brain, including both cortical and subcortical structures, and the stomatognathic system. The top-down control from the brain to the stomatognathic system, such as the

Correspondence: winzlin@ym.edu.tw

Department of Dentistry, School of Dentistry, National Yang-Ming University, No. 155, Sec. 2, Linong Street, Taipei 11221, Taiwan coordination between jaw motion and tongue movement, has been established $[1,6]$. However, what has remained unclear is whether input from peripheral structures, such as the sensory signals from the jaw and teeth, can likewise affect the brain. Aging is associated with a decline in both stomatognathic (e.g., tooth loss) [7] and brain functions (e.g., cognitive impairment or dementia) [8]. Therefore, the mechanisms underlying the brain-stomatognathic axis have emerged as critical issues in neuroscience as well as in orofacial and geriatric medicine.

Accumulating evidence suggests that cognitive decline may be associated with masticatory dysfunction [9-15]. The term 'cognitive decline' generally refers to the decreased cognitive abilities, including short-term and long-term memory, reasoning, and language abilities, which can be associated with normal aging or dementia 
[16]. 'Masticatory dysfunction', as an umbrella term, refers to a debilitating condition in which normal masticatory function is compromised due to structural factors (e.g., tooth loss) or functional factors (e.g., weaker biting force or poorer masticatory performance) [17]. The association between cognitive decline and masticatory dysfunction was highlighted in the famous Nun study, which revealed that the number of missing teeth was associated with an increased risk of dementia [18]. The conclusion was supported by recent meta-analytical findings [9] and evidence from clinical [19] and animal research [10]. These data all suggested a close association between cognitive decline and masticatory dysfunction. For the general public, it is tempting to think that improving masticatory function may be a new path for preventing or ameliorating cognitive decline in elderly individuals [2-5].

In this review, we argue that the current evidence has not provided adequate information regarding the mechanisms underlying the relationship between cognitive decline and masticatory dysfunction. These findings may suggest a stomatognathic-to-brain effect, i.e., poorer masticatory conditions predispose individuals to cognitive decline. However, the cause-and-effect relationship is unclear, and a gap remains between theoretical and clinical investigations. To assess this issue, we first systematically reviewed the recent evidence in the past five years (2012.10.15 2017.10.15) regarding the association between cognitive decline and masticatory dysfunction, focusing on the findings from clinical/epidemiological and animal research (for a comprehensive review of this topic based on earlier publications, please see $[15,20]$ ). The limitations of these research models will be discussed. Second, we reviewed the evidence from brain neuroimaging studies. We propose that neuroimaging would be an ideal tool for bridging the gap between clinical/epidemiological evidence and animal research. Third, we summarized and put forward three hypotheses regarding the mechanisms underlying the brainstomatognathic axis. These hypotheses can be tested using neuroimaging methods. Finally, we summarized the future directions for research into the brainstomatognathic axis.

\section{Evidence from clinical and epidemiological research}

First, we reviewed the findings from systematic reviews or meta-analyses published in the past five years regarding the association between cognitive decline and masticatory dysfunction (for detailed procedures of the literature search and screening, please see Additional file 1). As shown in Table 1, two studies supported for the notion that decreased masticatory function is associated with diminished cognitive functions [9, 11], whereas two studies concluded that the association remained 'inconclusive' or 'unclear' [12, 13]. Another study showed no statistically significant difference in the number of teeth between elderly individuals with and without dementia [14]. It should be noted that all five of the reviews stated some potential confounding effects or between-study heterogeneity in study design $[9,11-14]$ As a cause-and-effect relationship cannot be inferred from the findings of the cross-sectional studies [11, 13], we restrict our discussion to studies with a longitudinal, prospective design. In total, 13 prospective studies ([2133]) were reported on by the five reviews [9, 11-14]. Nine studies reported that a worse dental status was associated with cognitive decline $([22,25,27-29,32,33]$, one study showed a borderline significance of the association [31]), three studies reported no significant association $[24,26,30]$, and one reported a negative association, i.e., more missing teeth was associated with a lower risk of dementia [21]. Though the majority of the studies revealed an association between cognitive decline and masticatory dysfunction, a great heterogeneity existed in the outcome assessment regarding masticatory function. For example, the number of missing teeth was adopted as a primary index related to masticatory function [17]. In one study, the condition of tooth loss was categorized based on the number of teeth (fewer than 20 or not) [30], while another study assessed the number of missing teeth per decade of follow up [25].

Second, it is noteworthy that while most of the studies showed an association between tooth loss and cognitive decline, the elderly participants may have undergone long-term adaptation to their edentulousness/tooth-loss condition during chewing. Therefore, though being a feasible and reliable index, the number of teeth lost may not fully capture the change in masticatory function, and functional assessments (e.g., masticatory performance) may provide a better index for masticatory dysfunction [11]. To understand the association between masticatory performance and cognitive conditions, we performed a review by systematically searching for and screening the original studies that directly investigated the association between cognitive decline and masticatory ability using a masticatory performance test (Table 2). We found five studies published in the past five years that objectively quantified masticatory performance using functional assessments, including the color-changeable chewing gum test $[34,35]$, the twocolor chewing gum test [36,37], and the Optocal chewing test and the sieve fractionation test [38]. Masticatory performance decreased in patients with dementia compared to the controls $[36,38]$ and was associated with performance on cognitive tests [34, 35, 37] (Table 2). These findings suggested that functional assessments may be useful for assessing practical chewing performance. 


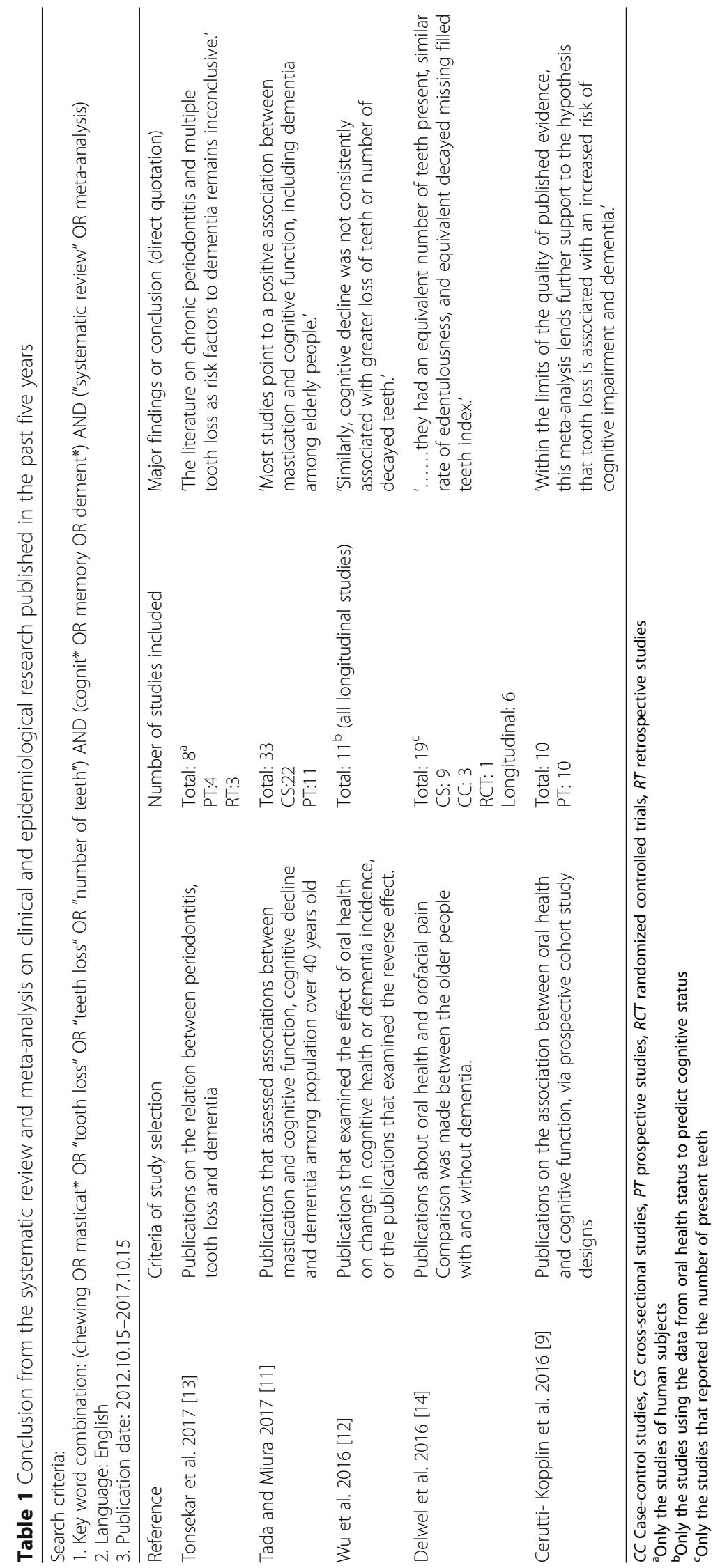




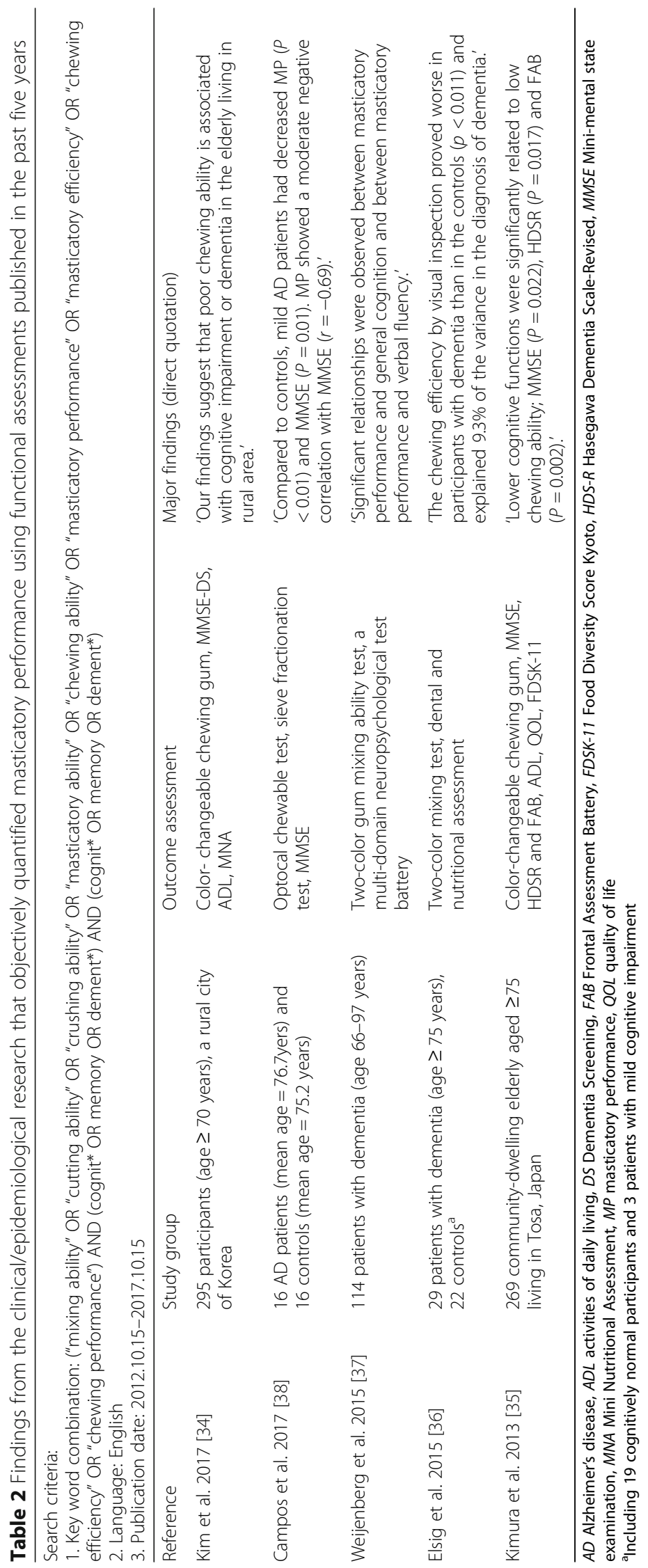


In general, the findings from recent reviews (Table 1) and studies of masticatory performance (Table 2) suggested that masticatory dysfunction was a potential risk factor for cognitive impairment in elderly individuals. However, these conclusions must be carefully interpreted due to the following limitations:

(A) As previously stated, there is a great heterogeneity among the methods for assessing masticatory function. In fact, only a small number of epidemiological surveys have directly assessed masticatory function, such as masticatory cutting ability, mixing ability or bite force, all of which represent different aspects of masticatory performance [17, 39]. Moreover, the self-reported chewing experience was not consistent with the results of masticatory performance assessments [40]. Therefore, it is questionable whether the number of teeth lost, a simplified epidemiological index of the dental condition, can fully capture the difficulty experienced during eating.

(B) Elderly individuals may develop some adaptive strategies to cope with tooth loss during eating. For example, they may carefully choose the type of food they eat and a method for processing it in order to more easily chew and swallow. Therefore, in regard to the association between cognitive decline and masticatory dysfunction, an individual's nutritional status, eating habits and general physical condition are critical factors to be adjusted for, especially in a longitudinal study [9].

(C) It is noteworthy that not all of the clinical/epidemiological studies included baseline or follow-up assessments of cognitive functions. Therefore, long-term changes in cognitive functions, which can be partly explained by normal aging, may not be adequately evaluated [16]. In addition, exercise is regarded as a critical factor to both physical fitness and cognitive ability in elderly individuals [41]. Long-term follow up on these factors would help to control for the effects of the baseline changes in general physical conditions.

\section{Evidence from animal research}

Compared to clinical and epidemiological studies, animal research provides benefits in examining the relevant mechanistic cause-effect relationships, e.g., the cellular or neurochemical changes associated with the studied behavioral deficits [42-60]. These findings from animal research published in the past five years are summarized in Table 3. In general, these studies have supported the hypothesis that cognitive decline is associated with masticatory dysfunction. These studies have demonstrated that cognitive decline is related to cellular and neurochemical change in the hippocampus, including decreased cellular proliferation [47, 48, 50], decreased levels of brain-derived neurotrophic factor [44, 47, 59], as well as increased nitrous oxide levels [55] and extracellular dopamine levels [52]. These findings suggested that the hippocampus-dependent deficits in learning and memory may contribute to the association between cognitive decline and masticatory dysfunction [10]. Importantly, using animal models, researchers were able to investigate the interactional effect between masticatory dysfunction and other factors, such as the type of diet [46, 59], environmental stimuli [47], and stress [52]. These factors can partially ameliorate the cognitive deficits induced by masticatory dysfunction [46, 47]. These findings imply that masticatory dysfunction per se may not be the only determinant to cognitive decline. Rather, the interaction of this dysfunction with other factors can partially account for the observed cognitive deficits.

Although animal research has provided a great deal of evidence regarding the mechanisms underlying the association between cognitive decline and masticatory dysfunction, several aspects need further clarification:

(A) One of the major challenges faced in interpreting these results is external validity, i.e., the extent to which we can generalize the findings from animal models to human subjects. Most of the animal studies adopted behavioral tasks, such as the Morris water maze and the passive avoidance task, which evaluated spatial and associative learning. One should bear in mind that a poor performance in these tasks does not necessarily reflect cognitive decline in elderly human subjects. The latter is a more complex condition, consisting of changes in short- and long-term memory, language, and reasoning.

(B) Most animal studies adopted tooth extraction as the experimental model to induce masticatory dysfunction, assuming that fewer molars leads to poorer chewing ability. In contrast, human subjects may develop adaptive strategies to cope with tooth loss. For instance, an earlier investigation on 315 edentulous elderly individuals showed that approximately $40 \%$ of them did not report chewing difficulty [61]. A recent investigation of elderly individuals revealed that chewing difficulty associated with a decreased number of functional units depended on the choice of food [62]. Notably, because experimental animals have a shorter lifespan, it may be difficult to evaluate the long-term adaptive effect of masticatory function using animal models.

\section{Bridging the gap: The role of brain neuroimaging in the investigation of the brain-stomatognathic axis}

Neuroimaging methods, including structural and functional magnetic resonance imaging (MRI), are popular approaches for investigating oral sensory and motor functions. The structural and functional brain signatures associated with cognitive decline, either due to normal aging or dementia, have been widely studied [16]. Brain neuroimaging provides the advantage of directly assessing the brain-stomatognathic axis by recording changes in functional activation and structural 


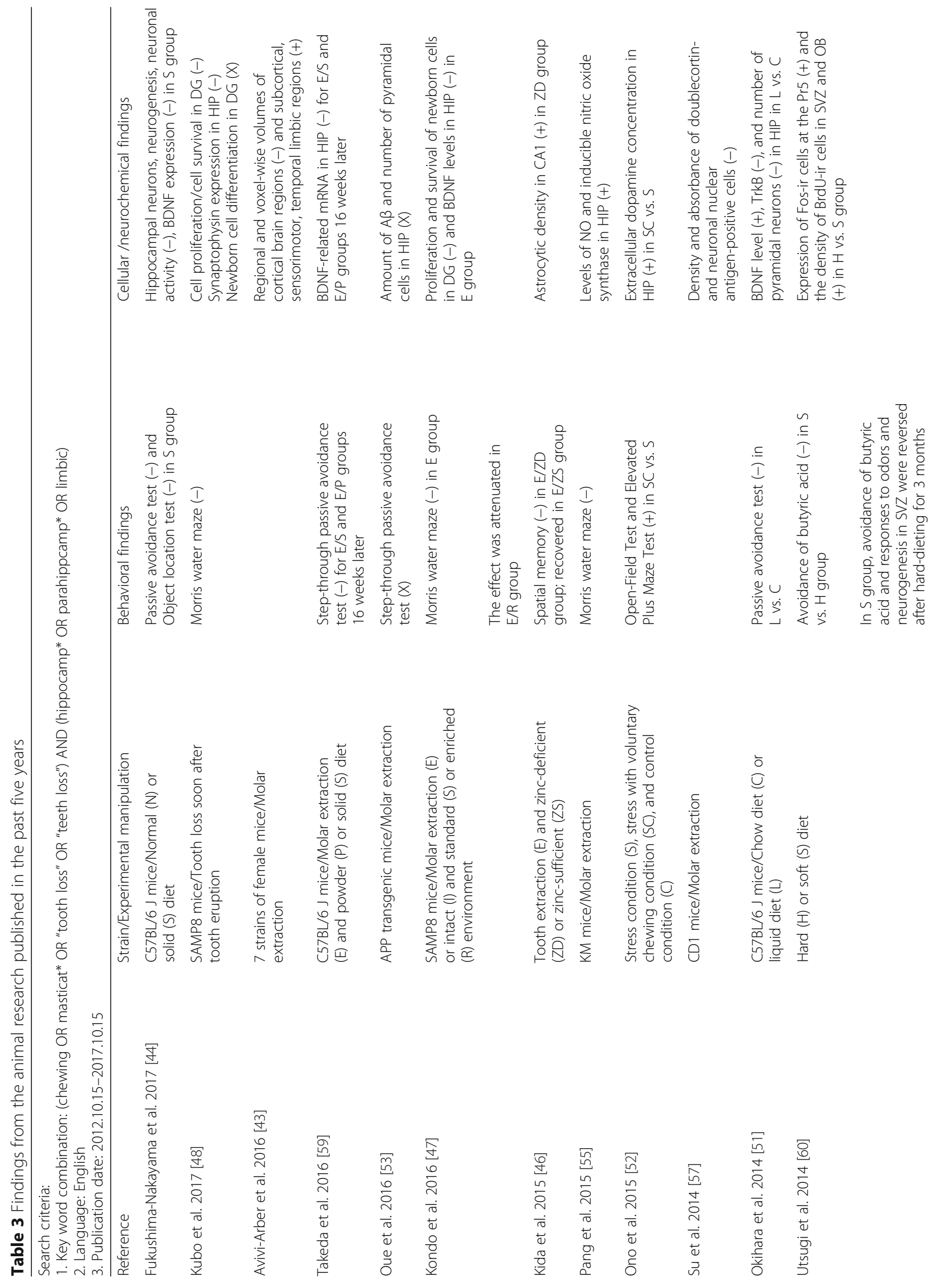




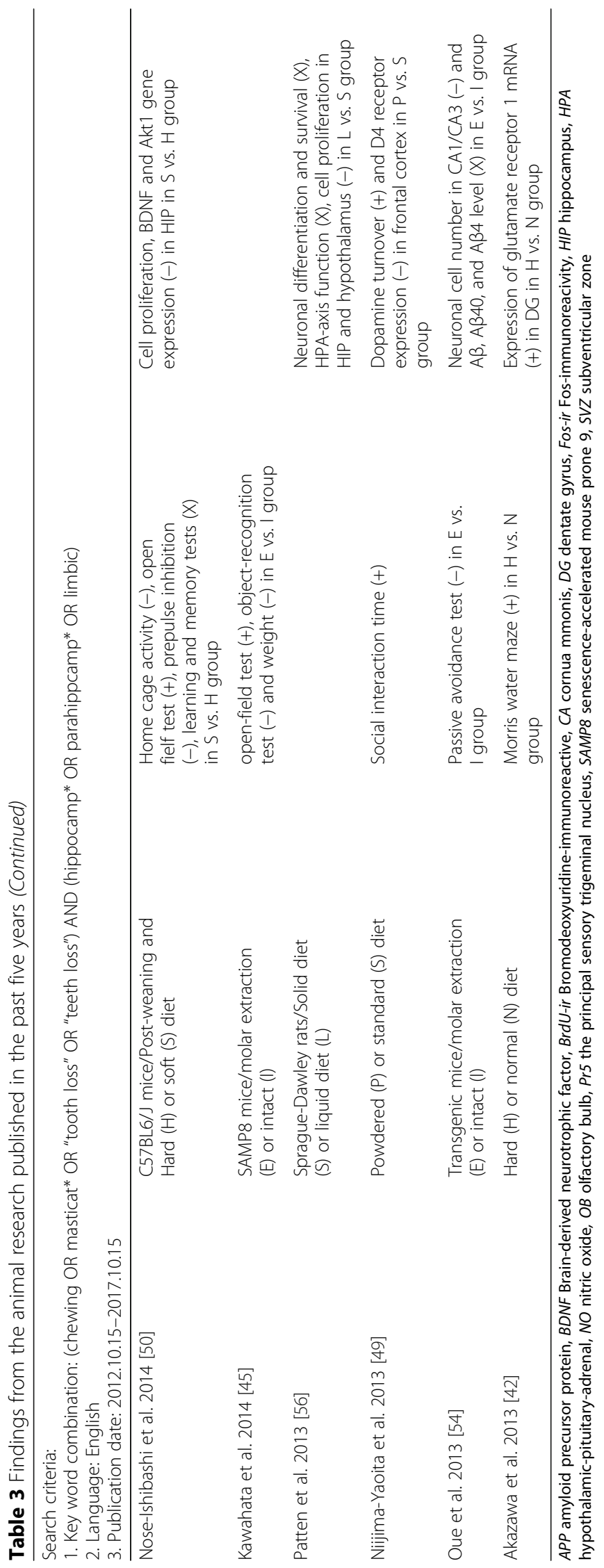


signatures. Therefore, these tools are suitable for testing hypotheses based on previous neuroimaging and animal research findings. Here, we propose three ways in which neuroimaging research may significantly contribute our knowledge of the brain-stomatognathic axis.

\section{Identifying the brain network associated with mastication}

In an earlier study, Onozuka and colleagues reported an age-related difference in chewing-related brain activation. These authors found a consistent activation pattern between older and younger groups [63]. Critically, the findings revealed a core network of mastication, including the sensorimotor cortex, the thalamus, the cerebellum, and the frontal cortex, including the supplementary motor area and the premotor cortex [63, 64]. In general, such a network was consistently reported by other studies that adopted similar gum-chewing tasks [65-70] (Table 4). Neuroimaging studies further elucidated that both blood perfusion of the trigeminal nucleus and brain activation in somatosensory areas were associated with chewing-side preference [66, 71]. Higher masticatory performance was also associated with the individual intrinsic brain signatures, including the greater gray matter volume in the motor area and higher functional connectivity between the cortex and the cerebellum [72]. Critically, neuroimaging research revealed that not only regional brain activation but also functional connectivity in mastication-related networks were associated with chewing [73]. Moreover, the difference in the connectional pattern was related to age [74]. In general, these findings implied that the functional and structural differences in mastication-related networks may play a key role in the individual variation in masticatory functions.

\section{Using brain imaging markers to explore the mechanisms underlying long-term changes in masticatory functions}

The mastication-related brain regions identified in the studies discussed above can be adopted as imaging markers for better elucidating the mechanisms underlying long-term stomatognathic behavior. First, neuroimaging research helps us to understand the individual responses to prosthodontic treatment [75]. Earlier neuroimaging studies showed that during a clenching task, brain activation in the somatosensory area was associated with the wearing of an implant-supported overdenture (IOD) but not with the wearing of a complete denture (CD) [76]. Moreover, during a gum-chewing task, brain activation in the prefrontal cortex (PFC) was reduced in the IOD group compared to the $\mathrm{CD}$ group [77]. Furthermore, compared to the conventional removable partial denture, the patients wearing a shortendental-arch denture showed no activation at the middle frontal gyrus, corresponding to lower masticatory performance [70]. Second, neuroimaging research helps to explore the mechanisms underlying long-term adaptation of treatment. For example, after denture insertion, brain activation in the somatosensory cortex was significantly increased during a jaw-clenching task, corresponding to an increase in chewing efficiency [68]. When the participants adapted to the insertion of an experimental palatal plate, there was a consistent increase in the activation of sensorimotor regions [78]. These findings showed that differences in brain mechanisms may play critical roles in the individual variation in long-term adaptation to treatment, particularly for psychological adaptation (i.e., incorporating the denture as 'part of the body' [68].

\section{Exploring the interaction between mastication and other cognitive-affective processing}

Evidence from behavioral research has revealed that chewing is associated with changes in sustained attention and helps relieve stress [79]. Evidence from animal research has consistently revealed that mastication attenuates activity in the hypothalamic-pituitary-adrenal axis and reduces the effect of chronic stress [80]. Here, neuroimaging may help to bridge the gap between human behavioral and animal research. For example, chewing has been associated with an increased activation in the attentional network when the participants performed an attention-demanding cognitive task [81]. In contrast, chewing may attenuate stress-induced activation of the salience network [82]. In contrast to animal research, a great number of cognitive-affective tasks can be implemented during MRI scans, providing a direct and in situ assessment of the association between mastication and cognition.

In the following section, several potential mechanisms and relevant hypotheses are summarized and discussed.

\section{Hypotheses regarding the neural mechanisms underlying the brain-stomatognathic axis and recent supporting evidence}

Current clinical / epidemiological findings have generally revealed a statistical correlation between cognitive decline and masticatory dysfunction. However, the neural mechanisms underlying this effect are still vague. Animal research has revealed that hippocampal deficits play a key role in this association. Based on the current evidence, we summarized and put forward several hypotheses for future neuroimaging studies (Fig. 1). These hypotheses are respectively focused on (a) the sensoryfeedback mechanisms of the stomatognathic system; (b) the compensatory control of movement from the brain; and (c) the role of the cerebellum, a brain region closely associated with sensorimotor and cognitive decline. 


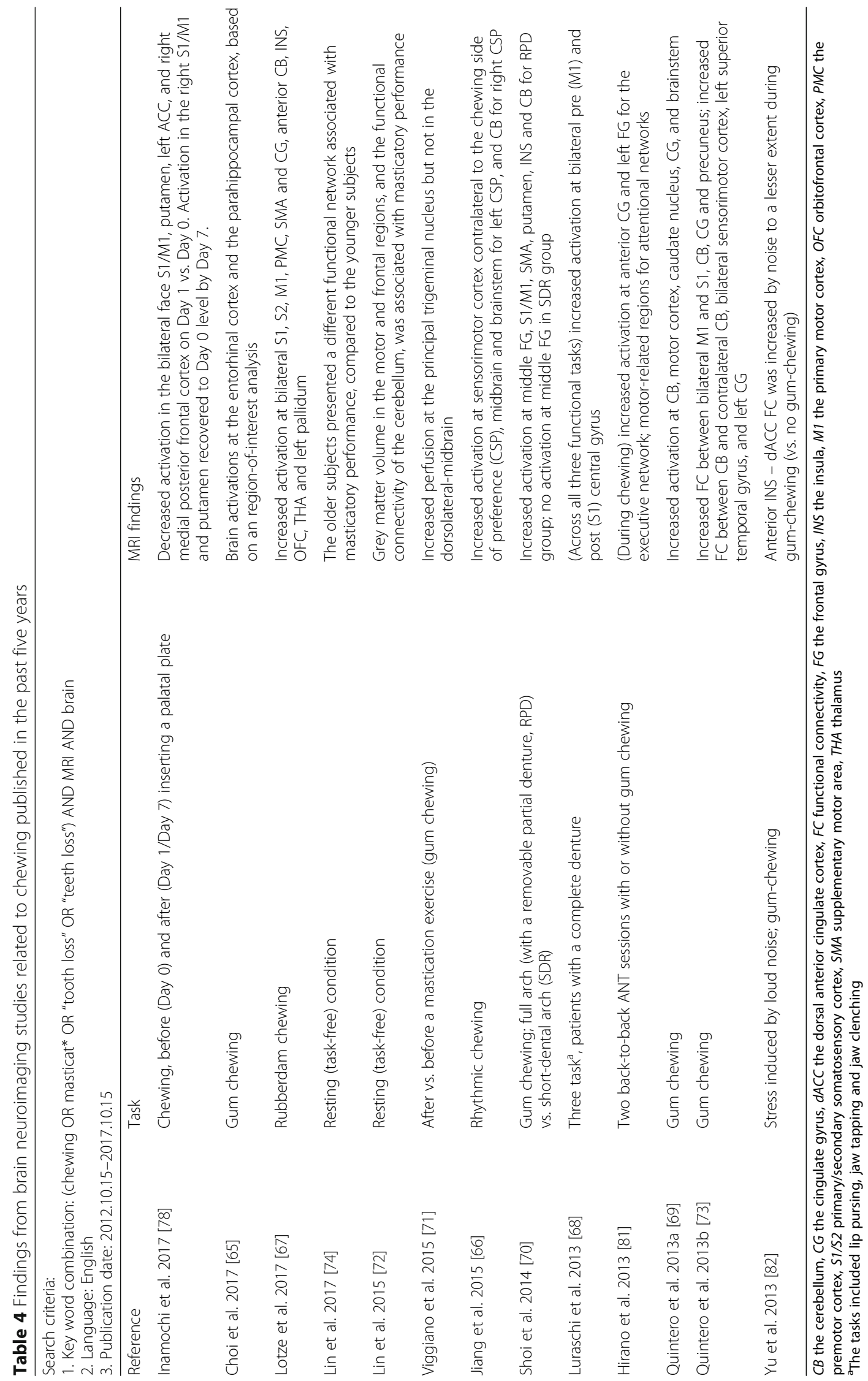



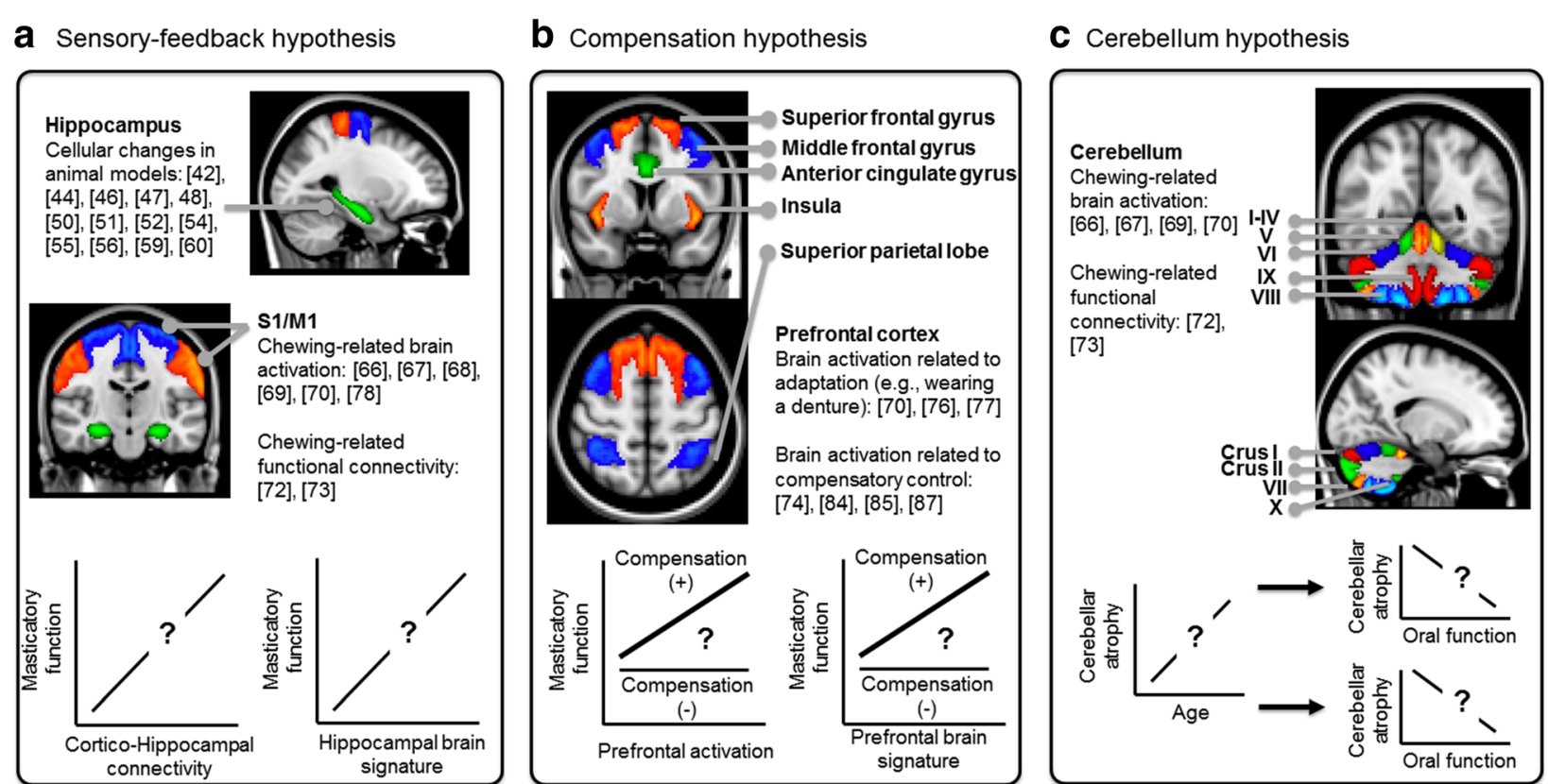

Fig. 1 The potential mechanisms underlying the brain-stomatognathic axis and the relevant hypotheses. a According to the sensory-feedback hypothesis, the cortico-hippocampal connectivity between the sensorimotor cortex (S1/M1) and the hippocampus, which deficits were observed in animal research, would be associated with the masticatory functions. b According to the compensation hypothesis, the age-related compensatory motor control, primarily mandated by the PFC, may be compromised in some elderly people. The deficits in the PFC and the network of cognitive control would be associated with both cognitive decline and masticatory dysfunction. c According to the cerebellum hypothesis, the cerebellar deficits would separately influence both motor and cognitive abilities. Therefore, cerebellar atrophy or decreased cerebellar function would be associated with both cognitive decline and masticatory dysfunction. The number in the bracket refers to the cited research evidence related to the hypotheses

\section{The sensory-feedback hypothesis}

Mastication may directly affect the brain via the sensorimotor circuitry (Fig. 1a). A potential mechanism is that the sensory feedback during mastication - primarily from the peripheral sensory apparatus - may stimulate the brain, with the hippocampus being a key target of this effect $[9,15]$. Supporting the hypothesis, neuroimaging evidence has consistently shown an increased activation in the sensorimotor area during a chewing task [66-70, 78] (Table 4). Increased brain activation in these regions is associated with the use of dentures, as shown by cross-sectional [70] and longitudinal research [68]. Furthermore, one recent study with a small sample size revealed that gum-chewing was associated with increased brain activation in the hippocampal / parahippocampal area [65], consistent with the findings from animal research [10]. Recent evidence has revealed a preferential connectivity between the hippocampus and the sensory cortex in mice. In contrast, a preferential connectivity between the hippocampus and the association cortex was found in humans subject [83]. These findings suggest that, sensory information can be rerouted to the association cortex before reaching the hippocampus in humans [83]. Critically, compared to younger participants, the older participants showed a stronger association between masticatory performance and intra-cortical connectivity [74]. These findings would suggest that intra-cortical and corticohippocampal connectivity, which signifies sensoryfeedback processing, is correlated with clinical metrics of masticatory functions - a hypothesis that remains untested with neuroimaging methods.

\section{The compensation hypothesis}

Accumulating evidence suggests that aging is associated with compensatory motor control processing, including increased monitoring as well as heightened attention and cognitive modulation [84, 85]. Such compensatory mechanisms are closely related to the brain regions that are associated with cognitive control and sensory integration, including the PFC, the parietal lobe, the insula, and the cingulate cortex $[84,85]$. The PFC showed a significant age-related atrophy [86], which plays a key role in cognitive decline [16]. Therefore, masticatory dysfunction may reflect a decreased capacity of compensation in motor control (Fig. 1b). The hypothesis was supported by neuroimaging findings from participants performed simple motor tasks. For example, younger individuals were able to maintain automatic and highly coordinated movement. In contrast, as age increased, the participants needed to pay more attention to monitoring and controlling their movement $[85,87]$. In terms of rhythmic 
movement, cortical connections are associated with a stronger effort in sensory integration and monitoring of motor control, while cerebellar connections are associated with an automatic modulation of movement [87]. A recent neuroimaging study revealed an age-related difference in the functional connectome of the mastication-related brain network. In the older participants, higher masticatory performance was positively correlated with the strength of intra-cortical and cortical-subcortical connectivity. In contrast, in the younger participants, high masticatory performance was positively correlated with connectivity between the somatosensory cortices and the cerebellum [74]. Neuroimaging evidence has consistently shown that wearing dentures is associated with increased brain activation in the PFC $[70,76]$. Wearing implant-supported dentures, which provide a better subjective adaptation than conventional dentures, was found to be associated with reduced activation in the PFC [77]. Together, these findings imply that when the PFC function is compromised, such as in the context of dementia, older individuals would exhibit poorer compensatory control over chewing movement, dampening their masticatory function.

\section{The cerebellum hypothesis}

The previous hypotheses presume that a direct association exists between cognitive decline and masticatory dysfunction. Alternatively, we reasoned that the decline in both cognitive and masticatory functions may be separately associated with deficits in other brain regions. We hypothesized that functional and structural changes in the cerebellum may play a key role to both cognitive decline and masticatory dysfunction in elderly individuals (Fig. 1c). Cerebellar atrophy is associated with poor cognitive performance [88] and predictive of the differentiation of Alzheimer's and Parkinson's dementia [89]. Cerebellar activation is frequently observed during chewing tasks $[63,66,69,70]$. The intrinsic functional connectivity of the cerebellum with motor regions was found to be associated with masticatory performance [72]. A recent study that compared the brain signatures between the physically frail and non-frail elderly individuals revealed that the gray matter volume in the cerebellum was associated with the motor-related index of physical frailty [90]. Atrophy of the cerebellar Crus I and Crus II (Fig. 1c) was observed to be specific to Alzheimer's disease and frontotemporal dementia [91]. Critically, the cerebellum plays a key role in age-related declines in cognitive and motor functions [92]. These findings suggest that a pre-existing deficit in critical brain regions, such as the cerebellum, may predispose individuals to functional declines in both mastication and cognition.

\section{Limitations: Beyond the brain-stomatognathic axis}

The abovementioned hypotheses focused on the brain mechanisms that underlie the brain-stomatognathic axis. We limited our search to recent studies that focused on the relevant brain and stomatognathic mechanisms. However, it should be noted that other factors may play key roles in the association between masticatory dysfunction and cognitive decline. (A) First, both tooth loss or poor masticatory performance are related to oral hygiene skills and behavior, which may be compromised in the elderly individuals with cognitive impairment [11]. (B) Second, nutritional biomarkers (e.g., cholesterol) were reported to be independent risk markers of cognitive decline [93], and in elderly individuals, masticatory ability may explain part of the variance in nutrient intake [94]. (C) Third, among the major causes of tooth loss are periodontal diseases, which are associated with inflammation related to periodontal pathogens. The inflammatory damage of small blood vessels may play a key role in the pathogenesis of Alzheimer's disease dementia [95]. Therefore, microbiological and immunological aspects, particularly periodontal conditions, should be considered [11, 13]. (D) Evidence from animal research has revealed that chewing may mediate the hippocampus-dependent cognitive deficit by suppressing HPA axis hyperactivity in the hippocampus [20, 80]. These considerations indicate that an integrative, multidisciplinary investigation -including behavioral, nutritional, immunological and hormonal research - is necessary for a full understanding of the brain-stomatognathic axis.

\section{Considerations for future research}

Based on the current evidence from the clinical, epidemiological, animal, and neuroimaging studies, we argue that the mechanisms underlying the brainstomatognathic axis have not been elucidated, and the cause-and-effect relationship between cognitive decline and masticatory dysfunction requires more investigation. We suggest that the following aspects of this field must be highlighted in future research:

\section{Refinement of behavioral assessments}

In terms of 'masticatory dysfunction' most recent studies focused only on the anatomical deficit of the stomatognathic system, using the number of missing teeth as an index. Again, the anatomical deficit does not necessarily reflect the subjective chewing experience or objective masticatory performance. A more comprehensive assessment of the specific elements of stomatognathic function (e.g., masticatory performance, biting force, oral stereognosis, and masticatory muscles) and their interaction should be considered in future research. In terms of 'cognitive decline', our review revealed a substantial gap 
between the cognitive abilities assessed in human subjects and those assessed in animal research. A better reconciliation of the assessments between different species would increase the validity of the conclusions drawn from animal research.

\section{Inclusion of baseline changes in mental and physical conditions}

As revealed by animal research, the effect of masticatory dysfunction on cognitive decline may interact with the nutritional $[46,59]$ and mental condition of the organism $[47,52]$. Notably, these factors are associated with the physical fitness of the elderly, and the interactional effect of these factors on cognitive decline should be considered.

\section{Experimental designs that include prospective, longitudinal observations}

One of the key requirements for understanding the mechanisms the brain-stomatognathic axis is the ability to differentiate between normal and pathological aging. The effect of long-term alterations, such as neuroplasticity effects in the brain, cannot be inferred from crosssectional findings. A systematic collection of the results from masticatory and cognitive assessments would help clarify the effect of normal aging.

\section{A precise estimation of the effect size}

From a clinical perspective, the critical question may not always be to determine 'If $\mathrm{X}$ and $\mathrm{Y}$ is associated'. Rather, to make a proper diagnosis and prognosis, clinicians need to know "To what extent does the change in X contribute the change in Y', i.e., an estimation of the effect size of the association. As shown in a recent metaanalysis, suboptimal dentition (i.e., having $<20$ teeth) was associated with a $20 \%$ higher risk of cognitive decline $[9,52]$. However, it is unclear if this is a pure effect from tooth loss or a combined effect due to other confounding factors. To estimate the actual effect that masticatory dysfunction has on cognitive decline is a clinically significant question.

\section{Conclusions}

By revisiting recent evidence regarding the association between cognitive decline and masticatory dysfunction, we argue that the mechanisms underlying the brainstomatognathic axis have not been fully elucidated. Moreover, it is concluded that the cause-and-effect relationship between cognitive decline and masticatory dysfunction requires more investigation. Three potential models and hypotheses of the brain-stomatognathic axis, focusing on the sensory feedback mechanisms, the compensation of motor control, and the cerebellum deficits, were summarized. Brain neuroimaging may contribute to identify the mechanisms underlying the brain-stomatognathic axis.

\section{Additional file}

Additional file 1: Detailed procedures for systematic review and a list of the articles included in the review. (DOCX $46 \mathrm{~kb}$ )

\section{Abbreviations}

APP: Amyloid precursor protein; BDNF: Brain-derived neurotrophic factor; CA: Cornu ammonis; CB: The cerebellum; CG: The cingulate gyrus; CS: Crosssectional studies; dACC: The dorsal anterior cingulate cortex; DG: Dentate gyrus; FC: Functional connectivity; FG: The frontal gyrus; HIP: Hippocampus; HPA: Hypothalamic-pituitary-adrenal; INS: The insula; M1: The primary motor cortex; MRI: Magnetic resonance imaging; OB: Olfactory bulb; PFC: The prefrontal cortex; PMC: The premotor cortex; Pr5: The principal sensory trigeminal nucleus; PT: Prospective studies; RT: Retrospective studies; S1/ S2: Primary/secondary somatosensory cortex; SMA: Supplementary motor area; SVZ: Subventricular zone

\section{Acknowledgments}

None.

Funding

This study was funded by the Ministry of Science and Technology of Taiwan (MOST 103-2314-B-010-025-MY3 and MOST 105-2628-B-010-008-MY4). The funding institution didn't influence the design of the study, collection, analysis, and interpretation of data, and preparation of the manuscript.

Availability of data and materials

Detailed procedures for systematic review and a list of the articles included in the review are included in Additional file 1.

Authors' contributions

CSL collected the articles for review, interpreted the results, and finalized the manuscript. The author read and approved the final manuscript.

Ethics approval and consent to participate

Not applicable.

Consent for publication

Not applicable.

\section{Competing interests}

The author declares no competing interests.

\section{Publisher's Note}

Springer Nature remains neutral with regard to jurisdictional claims in published maps and institutional affiliations.

Received: 30 June 2017 Accepted: 18 December 2017 Published online: 05 January 2018

\section{References}

1. Nelson SJ, Ash MM. Wheeler's dental anatomy, physiology, and occlusion. St. Louis, Missouri: Saunders, Elsevier Inc.; 2010

2. The Guardian: Chewing gum 'stops dementia'. 2000.

3. Lehrer J. The cognitive benefits of chewing gum. Wired; 2011.

4. News BBC. Chew your way to a better. Brain; 2002

5. Young E. Chewing gum improves memory. New Scientist; 2002.

6. Moore JD, Kleinfeld D, Wang F. How the brainstem controls orofacial behaviors comprised of rhythmic actions. Trends Neurosci. 2014:37(7):370-80

7. Kassebaum NJ, Bernabe E, Dahiya M, Bhandari B, Murray CJ, Marcenes W. Global burden of severe tooth loss: a systematic review and meta-analysis. J Dent Res. 2014;93(7 Suppl):20S-8S.

8. $\quad \mathrm{YT}$ W, Beiser AS, Breteler MMB, Fratiglioni L, Helmer C, Hendrie HC, Honda $\mathrm{H}$, Ikram MA, Langa KM, Lobo A, et al. The changing prevalence and incidence of dementia over time - current evidence. Nat Rev Neurol. 2017;

9. Cerutti-Kopplin D, Feine J, Padilha DM, de Souza RF, Ahmadi M, Rompre P, Booji L, Emami E. Tooth loss increases the risk of diminished cognitive 
function: a systematic review and meta-analysis. JDR Clinical \& Translational Research. 2016;

10. Chen $H$, linuma $M$, Onozuka $M$, Kubo KY. Chewing maintains hippocampusdependent cognitive function. Int J Med Sci. 2015;12(6):502-9.

11. Tada A, Miura H. Association between mastication and cognitive status: a systematic review. Arch Gerontol Geriatr. 2017;70:44-53.

12. Wu B, Fillenbaum GG, Plassman BL, Guo L. Association between oral health and cognitive status: a systematic review. J Am Geriatr Soc. 2016;64(4):739-51.

13. Tonsekar PP, Jiang SS, Yue G. Periodontal disease, tooth loss and dementia: is there a link? A systematic review. Gerodontology. 2017;34(2):151-63.

14. Delwel S, Binnekade TT, Perez RS, Hertogh CM, Scherder EJ, Lobbezoo F. Oral health and orofacial pain in older people with dementia: a systematic review with focus on dental hard tissues. Clin Oral Investig. 2017;21(1):17-32.

15. Weijenberg RA, Scherder EJ, Lobbezoo F. Mastication for the mind-the relationship between mastication and cognition in ageing and dementia. Neurosci Biobehav Rev. 2011;35(3):483-97.

16. Bishop NA, Lu T, Yankner BA. Neural mechanisms of ageing and cognitive decline. Nature. 2010;464(7288):529-35.

17. Ikebe K, Matsuda K, Kagawa R, Enoki K, Okada T, Yoshida M, Maeda Y. Masticatory performance in older subjects with varying degrees of tooth loss. J Dent. 2012:40(1):71-6.

18. Stein PS, Desrosiers M, Donegan SJ, Yepes JF, Kryscio RJ: Tooth losS, dementia and neuropathology in the nun study. J Am Dent Assoc 2007, 138(10):1314-1322; quiz 1381-1312.

19. Okamoto N, Morikawa M, Amano N, Yanagi M, Takasawa S, Kurumatani N. Effects of tooth loss and the apolipoprotein E varepsilon4 allele on mild memory impairment in the Fujiwara-kyo study of Japan: a nested casecontrol study. Journal of Alzheimer's disease : JAD. 2017;55(2):575-83.

20. Teixeira FB, Pereira Fernandes Lde M, Noronha PA, dos Santos MA, Gomes Leal W, Ferraz Maia Cdo S, Lima RR. Masticatory deficiency as a risk factor for cognitive dysfunction. Int J Med Sci. 2014;11(2):209-14.

21. Arrive E, Letenneur L, Matharan F, Laporte C, Helmer C, Barberger-Gateau P, Miquel $J$, Dartigues JF. Oral health condition of French elderly and risk of dementia: a longitudinal cohort study. Community Dent Oral Epidemiol. 2012;40(3):230-8.

22. Batty GD, Li Q, Huxley R, Zoungas S, Taylor BA, Neal B, de Galan B, Woodward M, Harrap SB, Colagiuri S, et al. Oral disease in relation to future risk of dementia and cognitive decline: prospective cohort study based on the action in diabetes and vascular disease: Preterax and Diamicron modified-release controlled evaluation (ADVANCE) trial. European Psychiatry J Assoc European Psychiatrists. 2013;28(1):49-52.

23. Chalmers JM, Carter KD, Spencer AJ. Oral diseases and conditions in community-living older adults with and without dementia. Special care in dentistry : official publication of the American Association of Hospital Dentists, the Academy of Dentistry for the Handicapped, and the American Society for Geriatric Dentistry. 2003;23(1):7-17.

24. Hansson P, Eriksson Sorman D, Bergdahl J, Bergdahl M, Nyberg L, Adolfsson R, Nilsson LG. Dental status is unrelated to risk of dementia: a 20-year prospective study. J Am Geriatr Soc. 2014;62(5):979-81.

25. Kaye EK, Valencia A, Baba N, Spiro A, 3rd, Dietrich T, Garcia Rl: Tooth loss and periodontal disease predict poor cognitive function in older men. J Am Geriatr Soc 2010, 58(4):713-718.

26. Naorungroj S, Schoenbach VJ, Wruck L, Mosley TH, Gottesman RF, Alonso A Heiss G, Beck J, Slade GD. Tooth loss, periodontal disease, and cognitive decline in the atherosclerosis risk in communities (ARIC) study. Community Dent Oral Epidemiol. 2015;43(1):47-57

27. Naorungroj S, Slade GD, Beck JD, Mosley TH, Gottesman RF, Alonso A, Heiss G. Cognitive decline and oral health in middle-aged adults in the ARIC study. J Dent Res. 2013;92(9):795-801.

28. Paganini-Hill A, White SC, Atchison KA. Dentition, dental health habits, and dementia: the leisure world cohort study. J Am Geriatr Soc. 2012;60(8):1556-63.

29. Reyes-Ortiz CA, Luque JS, Eriksson CK, Soto L. Self-reported tooth loss and cognitive function: data from the Hispanic established populations for epidemiologic studies of the elderly (Hispanic EPESE). Colombia medica. 2013;44(3):139-45.

30. Shimazaki Y, Soh I, Saito T, Yamashita Y, Koga T, Miyazaki H, Takehara T. Influence of dentition status on physical disability, mental impairment, and mortality in institutionalized elderly people. J Dent Res. 2001;80(1):340-5.
31. Stewart R, Weyant RJ, Garcia ME, Harris T, Launer LJ, Satterfield S, Simonsick EM, Yaffe K, Newman AB. Adverse oral health and cognitive decline: the health, aging and body composition study. J Am Geriatr Soc. 2013:61(2):177-84

32. Tsakos G, Watt RG, Rouxel PL, de Oliveira C, Demakakos P. Tooth loss associated with physical and cognitive decline in older adults. J Am Geriatr Soc. 2015;63(1):91-9.

33. Yamamoto T, Kondo K, Hirai H, Nakade M, Aida J, Hirata Y. Association between self-reported dental health status and onset of dementia: a 4-year prospective cohort study of older Japanese adults from the Aichi Gerontological evaluation study (AGES) project. Psychosom Med. 2012;74(3):241-8.

34. Kim EK, Lee SK, Choi YH, Tanaka M, Hirotsu K, Kim HC, Lee HK, Jung YS, Amano A. Relationship between chewing ability and cognitive impairment in the rural elderly. Arch Gerontol Geriatr. 2017;70:209-13.

35. Kimura Y, Ogawa H, Yoshihara A, Yamaga T, Takiguchi T, Wada T, Sakamoto $\mathrm{R}$, Ishimoto $\mathrm{Y}$, Fukutomi $\mathrm{E}$, Chen W, et al. Evaluation of chewing ability and its relationship with activities of daily living, depression, cognitive status and food intake in the community-dwelling elderly. Geriatr Gerontol Int 2013;13(3):718-25

36. Elsig F, Schimmel M, Duvernay E, Giannelli SV, Graf CE, Carlier S, Herrmann FR, Michel JP, Gold G, Zekry D, et al. Tooth loss, chewing efficiency and cognitive impairment in geriatric patients. Gerodontology. 2015:32(2):149-56.

37. Weijenberg RA, Lobbezoo F, Visscher CM, Scherder EJ. Oral mixing ability and cognition in elderly persons with dementia: a cross-sectional study. J Oral Rehabil. 2015;42(7):481-6.

38. Campos $\mathrm{CH}$, Ribeiro GR, Costa UL, Rodrigues Garcia RC. Correlation of cognitive and masticatory function in Alzheimer's disease. Clin Oral Investig. 2017;21(2):573-8.

39. Yamada A, Kanazawa M, Komagamine Y, Minakuchi S. Association between tongue and lip functions and masticatory performance in young dentate adults. J Oral Rehabil. 2015;42(11):833-9.

40. Carlsson GE, Lindquist LW. Ten-year longitudinal study of masticatory function in edentulous patients treated with fixed complete dentures on osseointegrated implants. Int J Prosthodont. 1994:7(5):448-53.

41. van Praag $\mathrm{H}$. Exercise and the brain: something to chew on. Trends Neurosci. 2009;32(5):283-90.

42. Akazawa Y, Kitamura T, Fujihara Y, Yoshimura Y, Mitome M, Hasegawa T. Forced mastication increases survival of adult neural stem cells in the hippocampal dentate gyrus. Int J Mol Med. 2013;31(2):307-14.

43. Avivi-Arber L, Seltzer Z, Friedel M, Lerch JP, Moayedi M, Davis KD, Sessle BJ. Widespread volumetric brain changes following tooth loss in female mice. Front Neuroanat. 2016:10:121.

44. Fukushima-Nakayama $Y$, Ono $T$, Hayashi $M$, Inoue $M$, Wake $H$, Ono $T$, Nakashima T. Reduced mastication impairs memory function. J Dent Res. 2017:96(9):1058-66.

45. Kawahata M, Ono Y, Ohno A, Kawamoto S, Kimoto K, Onozuka M. Loss of molars early in life develops behavioral lateralization and impairs hippocampus-dependent recognition memory. BMC Neurosci. 2014;15:4.

46. Kida K, Tsuji T, Tanaka S, Kogo M: Zinc deficiency with reduced mastication impairs spatial memory in young adult mice. Physiol Behav 2015, 152(Pt A):231-237.

47. Kondo H, Kurahashi M, Mori D, linuma M, Tamura Y, Mizutani K, Shimpo K, Sonoda S, Azuma K, Kubo KY. Hippocampus-dependent spatial memory impairment due to molar tooth loss is ameliorated by an enriched environment. Arch Oral Biol. 2016;61:1-7.

48. Kubo KY, Murabayashi C, Kotachi M, Suzuki A, Mori D, Sato Y, Onozuka M, Azuma K, linuma M. Tooth loss early in life suppresses neurogenesis and synaptophysin expression in the hippocampus and impairs learning in mice. Arch Oral Biol. 2017;74:21-7.

49. Niijima-Yaoita F, Tsuchiya M, Saito H, Nagasawa $Y$, Murai $S$, Arai $Y$, Nakagawasai O, Nemoto W, Tadano T, Tanno K. Influence of a long-term powdered diet on the social interaction test and dopaminergic systems in mice. Neurochem Int. 2013;63(4):309-15.

50. Nose-Ishibashi K, Watahiki J, Yamada K, Maekawa M, Watanabe A, Yamamoto G, Enomoto A, Matsuba Y, Nampo T, Taguchi T, et al. Soft-diet feeding after weaning affects behavior in mice: potential increase in vulnerability to mental disorders. Neuroscience. 2014;263:257-68.

51. Okihara H, Ito J, Kokai S, Ishida T, Hiranuma M, Kato C, Yabushita T, Ishida K, Ono T, Michikawa M. Liquid diet induces memory impairment accompanied 
by a decreased number of hippocampal neurons in mice. J Neurosci Res. 2014:92(8):1010-7.

52. Ono Y, Koizumi S, Onozuka M. Chewing prevents stress-induced hippocampal LTD formation and anxiety-related behaviors: a possible role of the dopaminergic system. Biomed Res Int. 2015;2015:294068.

53. Oue H, Miyamoto Y, Koretake K, Okada S, Doi K, Jung CG, Michikawa M, Akagawa Y. Tooth loss might not alter molecular pathogenesis in an aged transgenic Alzheimer's disease model mouse. Gerodontology. 2016:33(3):308-14.

54. Oue H, Miyamoto Y, Okada S, Koretake K, Jung CG, Michikawa M, Akagawa Y. Tooth loss induces memory impairment and neuronal cell loss in APP transgenic mice. Behav Brain Res. 2013;252:318-25.

55. Pang Q, Hu X, Li X, Zhang J, Jiang Q. Behavioral impairments and changes of nitric oxide and inducible nitric oxide synthase in the brains of molarless KM mice. Behav Brain Res. 2015;278:411-6.

56. Patten AR, Moller DJ, Graham J, Gil-Mohapel J, Christie BR. Liquid diets reduce cell proliferation but not neurogenesis in the adult rat hippocampus. Neuroscience. 2013;254:173-84.

57. Su S, Qi T, Su B, Gu H, Wang J, Yang L. Tooth loss inhibits neurogenesis in the dentate gyrus of adult mice. Neural Regen Res. 2014;9(17):1606-9.

58. Suzuki A, linuma M, Hayashi S, Sato Y, Azuma K, Kubo KY. Maternal chewing during prenatal stress ameliorates stress-induced hypomyelination, synaptic alterations, and learning impairment in mouse offspring. Brain Res. 2016;1651:36-43

59. Takeda Y, Oue H, Okada S, Kawano A, Koretake K, Michikawa M, Akagawa Y, Tsuga K. Molar loss and powder diet leads to memory deficit and modifies the mRNA expression of brain-derived neurotrophic factor in the hippocampus of adult mice. BMC Neurosci. 2016;17(1):81.

60. Utsugi C, Miyazono S, Osada K, Sasajima H, Noguchi T, Matsuda M, Kashiwayanagi M. Hard-diet feeding recovers neurogenesis in the subventricular zone and olfactory functions of mice impaired by soft-diet feeding. PLoS One. 2014;9(5):e97309.

61. Slade GD, Spencer AJ, Roberts-Thomson K. Tooth loss and chewing capacity among older adults in Adelaide. Aust N Z J Public Health. 1996;20(1):76-82.

62. Hsu KJ, Lee HE, Lan SJ, Huang ST, Chen CM, Yen YY. Evaluation of a selfassessed screening test for masticatory ability of Taiwanese older adults. Gerodontology. 2012;29(2):e1113-20.

63. Onozuka M, Fujita M, Watanabe K, Hirano Y, Niwa M, Nishiyama K, Saito S. Age-related changes in brain regional activity during chewing: a functional magnetic resonance imaging study. J Dent Res. 2003:82(8):657-60.

64. Onozuka M, Fujita M, Watanabe K, Hirano Y, Niwa M, Nishiyama K, Saito S. Mapping brain region activity during chewing: a functional magnetic resonance imaging study. J Dent Res. 2002;81(11):743-6.

65. Choi YH, Jang WH, Im SU, Song KB, Lee HK, Lee HD, Seo YS, Jang SH. The brain activation pattern of the medial temporal lobe during chewing gum: a functional MRI study. Neural Regen Res. 2017;12(5):812-4.

66. Jiang H, Liu H, Liu G, Jin Z, Wang L, Ma J, Li H. Analysis of brain activity involved in chewing-side preference during chewing: an fMRI study. J Oral Rehabil. 2015:42(1):27-33

67. Lotze M, Domin M, Kordass B. Symmetry of fMRI activation in the primary sensorimotor cortex during unilateral chewing. Clin Oral Investig. 2017;21(4):967-73.

68. Luraschi J, Korgaonkar MS, Whittle T, Schimmel M, Muller F, Klineberg I. Neuroplasticity in the adaptation to prosthodontic treatment. J Orofac Pain. 2013;27(3):206-16.

69. Quintero A, Ichesco E, Myers C, Schutt R, Gerstner GE. Brain activity and human unilateral chewing: an FMRI study. J Dent Res. 2013;92(2):136-42.

70. Shoi K, Fueki K, Usui N, Taira M, Wakabayashi N. Influence of posterior dental arch length on brain activity during chewing in patients with mandibular distal extension removable partial dentures. J Oral Rehabil. 2014;41(7):486-95.

71. Viggiano A, Manara R, Conforti R, Paccone A, Secondulfo C, Lorusso L, Sbordone L, Di Salle F, Monda M, Tedeschi G, et al. Mastication induces long-term increases in blood perfusion of the trigeminal principal nucleus. Neuroscience. 2015:311:75-80.

72. Lin CS, SY W, CY W, Ko HW. Gray matter volume and resting-state functional connectivity of the motor cortex-cerebellum network reflect the individual variation in masticatory performance in healthy elderly people. Front Aging Neurosci. 2015;7:247.

73. Quintero A, Ichesco E, Schutt R, Myers C, Peltier S, Gerstner GE. Functional connectivity of human chewing: an fcMRI study. J Dent Res. 2013;92(3):272-8.
74. Lin CS, CY W, SY W, Lin HH, Cheng DH, Lo WL. Age-related difference in functional brain connectivity of mastication. Front Aging Neurosci. 2017;9:82.

75. Ohkubo C, Morokuma M, Yoneyama Y, Matsuda R, Lee JS. Interactions between occlusion and human brain function activities. J Oral Rehabil. 2013;40(2):119-29.

76. Yan C, Ye L, Zhen J, Ke L, Gang L. Neuroplasticity of edentulous patients with implant-supported full dentures. Eur J Oral Sci. 2008;116(5):387-93.

77. Kimoto K, Ono Y, Tachibana A, Hirano Y, Otsuka T, Ohno A, Yamaya K, Obata T, Onozuka M. Chewing-induced regional brain activity in edentulous patients who received mandibular implant-supported overdentures: a preliminary report. Journal of prosthodontic research. 2011;55(2):89-97.

78. Inamochi Y, Fueki K, Usui N, Taira M, Wakabayashi N. Adaptive change in chewing-related brain activity while wearing a palatal plate: an functional magnetic resonance imaging study. J Oral Rehabil. 2017:44(10):770-8.

79. Hirano Y, Onozuka M. Chewing and attention: a positive effect on sustained attention. Biomed Res Int. 2015;2015:367026.

80. Azuma K, Zhou Q, Niwa M, Kubo KY. Association between mastication, the hippocampus, and the HPA Axis: a comprehensive review. Int J Mol Sci. 2017;18(8)

81. Hirano Y, Obata T, Takahashi H, Tachibana A, Kuroiwa D, Takahashi T, Ikehira $\mathrm{H}$, Onozuka M. Effects of chewing on cognitive processing speed. Brain Cogn. 2013;81(3):376-81.

82. Yu H, Chen X, Liu J, Zhou X. Gum chewing inhibits the sensory processing and the propagation of stress-related information in a brain network. PLoS One. 2013:8(4):e57111.

83. Bergmann E, Zur G, Bershadsky G, Kahn I. The Organization of Mouse and Human Cortico-Hippocampal Networks Estimated by intrinsic functional connectivity. Cereb Cortex. 2016;26(12):4497-512.

84. Ward NS. Compensatory mechanisms in the aging motor system. Ageing Res Rev. 2006;5(3):239-54.

85. Seidler RD, Bernard JA, Burutolu TB, Fling BW, Gordon MT, Gwin JT, Kwak Y, Lipps DB. Motor control and aging: links to age-related brain structural, functional, and biochemical effects. Neurosci Biobehav Rev. 2010;34(5):721-33

86. Hutton C, Draganski B, Ashburner J, Weiskopf N. A comparison between voxel-based cortical thickness and voxel-based morphometry in normal aging. Neurolmage. 2009;48(2):371-80.

87. Heuninckx S, Wenderoth N, Debaere F, Peeters R, Swinnen SP. Neural basis of aging: the penetration of cognition into action control. J Neuroscience : Official J Soc Neuroscience. 2005;25(29):6787-96.

88. Thomann PA, Schlafer C, Seidl U, Santos VD, Essig M, Schroder J. The cerebellum in mild cognitive impairment and Alzheimer's disease - a structural MRI study. J Psychiatr Res. 2008;42(14):1198-202.

89. Persson K, Selbaek G, Braekhus A, Beyer M, Barca M, Engedal K. Fully automated structural MRI of the brain in clinical dementia workup. Acta Radiol. 2017;58(6):740-7.

90. Chen WT, Chou KH, Liu LK, Lee PL, Lee WJ, Chen LK, Wang PN, Lin CP. Reduced cerebellar gray matter is a neural signature of physical frailty. Hum Brain Mapp. 2015;36(9):3666-76.

91. Gellersen HM, Guo CC, O'Callaghan C, Tan RH, Sami S, Hornberger M. Cerebellar atrophy in neurodegeneration-a meta-analysis. J Neurol Neurosurg Psychiatry. 2017;

92. Bernard JA, Seidler RD. Moving forward: age effects on the cerebellum underlie cognitive and motor declines. Neurosci Biobehav Rev. 2014;42:193-207.

93. Taniguchi Y, Shinkai S, Nishi M, Murayama H, Nofuji Y, Yoshida H, Fujiwara Y. Nutritional biomarkers and subsequent cognitive decline among community-dwelling older Japanese: a prospective study. J Gerontology Series A, Biological sciences and medical sciences. 2014;69(10):1276-83.

94. Tada A, Miura H. Systematic review of the association of mastication with food and nutrient intake in the independent elderly. Arch Gerontol Geriatr. 2014:59(3):497-505.

95. Marchesi VT. Alzheimer's dementia begins as a disease of small blood vessels, damaged by oxidative-induced inflammation and dysregulated amyloid metabolism: implications for early detection and therapy. FASEB journal : official publication of the Federation of American Societies for Experimental Biology. 2011;25(1):5-13. 